\title{
Fly Ash Utilisation in Mullite Fabrication: Development of Novel Percolated Mullite
}

\author{
Pramod Koshy ${ }^{1, * \mathbb{D}}$, Naomi Ho ${ }^{1}$, Vicki Zhong ${ }^{1}$, Luisa Schreck ${ }^{1}$, Sandor Alex Koszo ${ }^{2}$, Erik J. Severin ${ }^{2}$ \\ and Charles Christopher Sorrell ${ }^{1}$
}

1 School of Materials Science and Engineering, UNSW Sydney, Sydney, NSW 2052, Australia; naomi.ho@unsw.edu.au (N.H.); vicki.zhong@unsw.edu.au (V.Z.); luisa.schreck@yahoo.de (L.S.); C.Sorrell@unsw.edu.au (C.C.S.)

2 Vecor Technologies Pty. Ltd., Sydney, NSW 2039, Australia; sandorkoszo@gmail.com (S.A.K.); e.severin@vecortech.com (E.J.S.)

* Correspondence: koshy@unsw.edu.au

\section{check for}

updates

Citation: Koshy, P.; Ho, N.; Zhong, V.; Schreck, L.; Koszo, S.A.; Severin, E.J.; Sorrell, C.C. Fly Ash Utilisation in Mullite Fabrication: Development of Novel Percolated Mullite. Minerals 2021, 11, 84. https://doi.org/ $10.3390 / \min 11010084$

Received: 18 December 2020

Accepted: 11 January 2021

Published: 16 January 2021

Publisher's Note: MDPI stays neutra with regard to jurisdictional claims in published maps and institutional affiliations.

Copyright: (c) 2021 by the authors. Licensee MDPI, Basel, Switzerland. This article is an open access article distributed under the terms and conditions of the Creative Commons Attribution (CC BY) license (https:/ / creativecommons.org/licenses/by/ $4.0 /)$.

\begin{abstract}
Fly ash is an aluminosilicate and the major by-product from coal combustion in power stations; its increasing volumes are major economic and environmental concerns, particularly since it is one of the largest mineral resources based on current estimates. Mullite $\left(3 \mathrm{Al}_{2} \mathrm{O}_{3} \cdot 2 \mathrm{SiO}_{2}\right)$ is the only stable phase in the $\mathrm{Al}_{2} \mathrm{O}_{3}-\mathrm{SiO}_{2}$ system and is used in numerous applications owing to its high-temperature chemical and mechanical stabilities. Hence, fly ash offers a potential economical resource for mullite fabrication, which is confirmed by a review of the current literature. This review details the methodologies to utilise fly ash with different additives to fabricate what are described as porous interconnected mullite skeletons or dense mullite bodies of approximately stoichiometric compositions. However, studies of pure fly ash examined only high- $\mathrm{Al}_{2} \mathrm{O}_{3}$ forms and none of these works reported long-term, high-temperature, firing shrinkage data for these mullite bodies. In the present work, high- $\mathrm{SiO}_{2}$ fly ashes were used to fabricate percolated mullite, which is demonstrated by the absence of firing shrinkage upon long-term high-temperature soaking. The major glass component of the fly ash provides viscosities suitably high for shape retention but low enough for ionic diffusion and the minor mullite component provides the nucleating agent to grow mullite needles into a direct-bonded, single-crystal, continuous, needle network that prevents high-temperature deformation and isolates the residual glass in the triple points. These attributes confer outstanding long-term dimensional stability at temperatures exceeding $1500{ }^{\circ} \mathrm{C}$, which is unprecedented for mullite-based compositions.
\end{abstract}

Keywords: fly ash; mullite; high temperature; percolation; long-term heating

\section{Introduction}

Owing to rapid industrialisation, there has been an increase in power generation, with numerous coal-powered electricity stations being used currently at high to maximal capacities. This has resulted in increased amounts of by-products being produced from the coal combustion process in these power stations; these products include fly ash, cenospheres, and bottom ash [1]. Of these, there has been increasing focus on the utilisation of fly ash since it is the major component, which is collected in electrostatic precipitators [2]. Recent estimates indicate that the annual global production of fly ash is $~ 800 \mathrm{mT}$ [3]. Unused fly ash generally is stored on-site at power stations in containers or in a slurry form in collection ponds [4]. The former strategy creates problems in terms of space requirements for storage while the latter can be subject to leaching of toxic elements from the fly ash [4]. Dumping of fly ash into water resources is known to occur in some countries, leading to major environmental issues [5]. These volumes illustrate the need for novel strategies for the utilisation of fly ash since only $20-50 \%$ is used commercially [3], with the major application being for the cement industry as pozzolan/aggregate [6]. However, higher 
utilisation in some European countries is the case as they produce very low volumes, although the utilisation levels by major producers in Asia and North America are low [3].

Fly ash consists of whitish spherical particles of size in the range 1-200 $\mu \mathrm{m}$, with average sizes being 20-40 $\mu \mathrm{m}$ [4]. Fly ash may contain remnants of unburnt carbon from the coal, which makes it appear grey to black in colour. Moreover, it may contain varying amounts of iron oxide, which can generate colours ranging from light to dark brown [4]. The proportions of the different oxides in fly ash vary with the source of the coal and the combustion process. On the basis of composition, the major oxide components are $\mathrm{Al}_{2} \mathrm{O}_{3}$ and $\mathrm{SiO}_{2}$, which result from the fact that clay and sand are the principal mineral phases of coal. While $\mathrm{SiO}_{2}$ is the major component of the glassy spherical particles, undissolved $\alpha$-quartz $\left(\mathrm{SiO}_{2}\right)$ generally is the principal crystalline phase. Another significant crystalline species that form during coal combustion is mullite $\left(3 \mathrm{Al}_{2} \mathrm{O}_{3} \cdot 2 \mathrm{SiO}_{2}\right)$ while lower levels of magnetite $\left(\mathrm{Fe}_{3} \mathrm{O}_{4}\right)$ and /or hematite $\left(\mathrm{Fe}_{2} \mathrm{O}_{3}\right)$ also are observed commonly [7]. While Class $\mathrm{F}$ fly ashes are low in $\mathrm{CaO}$, Class $\mathrm{C}$ fly ashes have higher $\mathrm{CaO}$ contents [8], which have been observed by the authors to be $>10 \mathrm{wt} \% \mathrm{CaO}$. Mullite is generally seen as a reaction product from reactions of $\mathrm{Al}_{2} \mathrm{O}_{3}$ - and $\mathrm{SiO}_{2}$-containing materials [9].

The widespread presence of mullite in fly ash suggests the applicability of such fly ashes as sources of mullite-based products. High-temperature applications are suggested by mullite's high melting point of $1850{ }^{\circ} \mathrm{C}$, as shown in the $\mathrm{Al}_{2} \mathrm{O}_{3}-\mathrm{SiO}_{2}$ phase diagram of Figure 1a [10]. Other advantageous attributes of mullite are its stability in both oxidising and reducing atmospheres [11]; moderate coefficient of thermal expansion, which generally is in the range $\alpha_{25-1000}=\sim 4.5-6.0 \times 10^{-6}{ }^{\circ} \mathrm{C}^{-1}$ [12]; good thermal shock and spalling resistance; high shear modulus; low thermal conductivity; good resistance to acidic and neutral slags; and excellent general corrosion resistance.
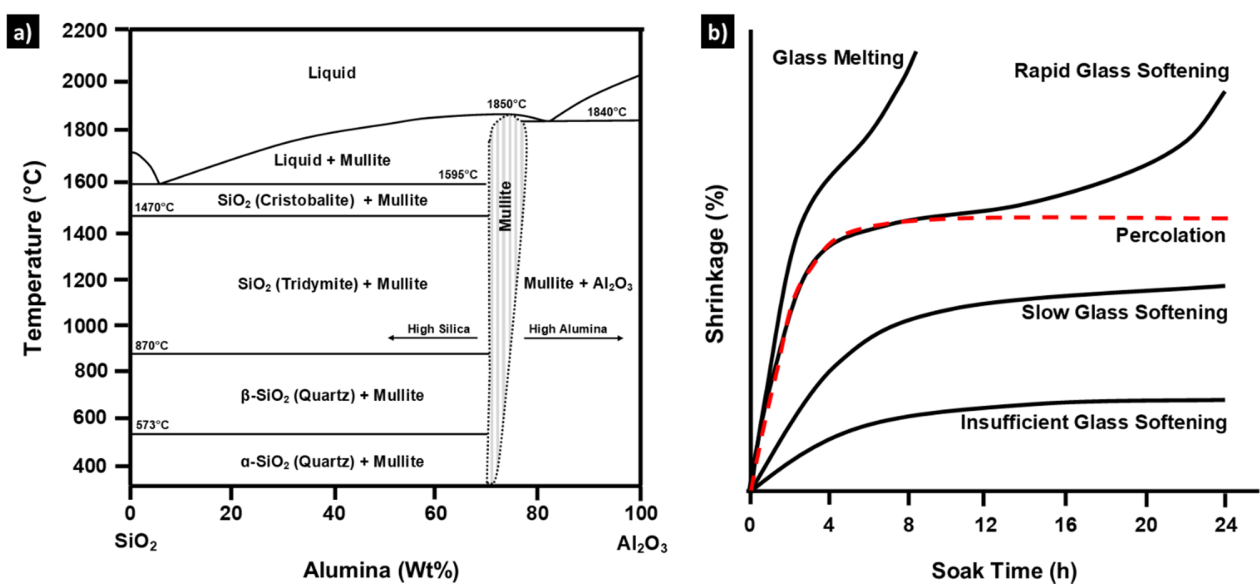

Figure 1. (a) $\mathrm{Al}_{2} \mathrm{O}_{3}-\mathrm{SiO}_{2}$ phase diagram, assuming congruently melting mullite (adapted from [10]) and (b) typical firing shrinkage curves for refractory products on sintering at high temperatures.

However, the applicability of fly ash as a source of mullite-based refractory products is challenged by its high glass content, the softening of which is well known as the principal source of high-temperature deformation of many oxide ceramics [13]. As shown in Figure $1 \mathrm{~b}$, the heating of highly vitreous bodies such as fly ash, which generally contain $\sim 60-70 \mathrm{wt} \%$ glass [14], would be expected to result in sigmoidal firing shrinkage kinetics. That is, initial slow glass softening at high glass viscosities would enhance particle rearrangement and firing shrinkage, followed by slumping owing to rapid deformation at low glass viscosities.

As suggested in Figure 1a, the stoichiometry of mullite ranges from an $\mathrm{Al}_{2} \mathrm{O}_{3}: \mathrm{SiO}_{2}$ molar ratio of 3:2 to 2:1 [9,12]. Single-crystal mullite is observed in the form of acicular (needlelike) or equiaxed grains, the morphologies of which vary with the $\mathrm{Al}_{2} \mathrm{O}_{3}: \mathrm{SiO}_{2}$ ratio, with lower $\mathrm{Al}_{2} \mathrm{O}_{3}$ contents' (viz., 3:2 mullite) resulting in more anisotropic grains [12]. Further, the morphology is affected by the addition of oxides (e.g., $\mathrm{Fe}_{2} \mathrm{O}_{3}$ enhances anisotropy [12]), 
which may be related to the effect of the additive on the glass viscosity, while anisotropy is restrained by high glass viscosities from low-fluxing additives (e.g., $\mathrm{Cr}_{2} \mathrm{O}_{3}$ [15]). While mullite is of the orthorhombic crystal structure, the preferred growth direction of mullite needles is [001] [16-18]. Although there are several reports of high-temperature axial thermal expansions, interpolation of the most comprehensive set of data [19] for 3:2 mullite gives the following axial and bulk coefficients of thermal expansion:

- $a$-axis: $\alpha_{20-1000}=5.39 \times 10^{-6}{ }^{\circ} \mathrm{C}^{-1}$

- b-axis: $\alpha_{20-1000}=7.73 \times 10^{-6}{ }^{\circ} \mathrm{C}^{-1}$

- $\quad c$-axis: $\alpha_{20-1000}=7.10 \times 10^{-6}{ }^{\circ} \mathrm{C}^{-1}$

- Bulk: $\alpha_{20-1000}=6.74 \times 10^{-6}{ }^{\circ} \mathrm{C}^{-1}$

Although mullite-containing materials are used commonly as commercial refractory products, the mineral mullite is not available as a bulk raw material for refractory fabrication [20]. Consequently, mullite generally is produced by heat treatment of other aluminosilicates, including kaolinite $\left(\mathrm{Al}_{2} \mathrm{O}_{3} \cdot 2 \mathrm{SiO}_{2} \cdot 2 \mathrm{H}_{2} \mathrm{O}\right)$, andalusite/sillimanite/kyanite $\left(\mathrm{Al}_{2} \mathrm{O}_{3} \cdot \mathrm{SiO}_{2}\right)$, or pyrophyllite $\left(4 \mathrm{SiO}_{2} \cdot \mathrm{Al}_{2} \mathrm{O}_{3} \cdot \mathrm{H}_{2} \mathrm{O}\right)$ at temperatures $\geq 1200{ }^{\circ} \mathrm{C}$, although these products consist of glass-bonded aggregates [12]. The different mullite-based refractory products are classified according to their $\mathrm{Al}_{2} \mathrm{O}_{3}$ contents and related pyrometric cone equivalents (PCEs) into fireclay $\left(\sim 25-45 \mathrm{wt} \% \mathrm{Al}_{2} \mathrm{O}_{3} ; \mathrm{PCE}=31-35\right)$, aluminous $(\sim 45-65 \mathrm{wt} \%$ $\left.\mathrm{Al}_{2} \mathrm{O}_{3} ; \mathrm{PCE}=35-38\right)$, and mullite $\left(\sim 65-75 \mathrm{wt} \% \mathrm{Al}_{2} \mathrm{O}_{3} ; \mathrm{PCE}=38-39\right)$ products $[20,21]$.

The refractoriness of mullite in principle is rated at $1850^{\circ}-1865^{\circ} \mathrm{C}(\mathrm{PCE}=38-39)$ [22] but the actual performance is somewhat lower owing to glass softening and resultant firing shrinkage. In practice, pure single-phase mullite exhibits short-term creep resistance at temperatures $\leq 1400{ }^{\circ} \mathrm{C}$ and commercial mullites perform similarly only to $\leq 1000{ }^{\circ} \mathrm{C}$ [13]. This degradation in practice highlights the importance of the achievement of direct bonding between the mullite grains in contrast to chemically (glass) bonded refractories, the thermal properties of which are dominated by the glassy matrix [23].

While the thermal resistance is a critical factor in refractory performance, chemical resistance often is an essential quality in metallurgical applications. Mullite-containing refractories are used commonly as linings for molten aluminium-alloy holding furnaces [24]. In these refractories, the molten metal wets and corrodes the refractory, which leads to the reduction of the siliceous component of mullite and the resultant formation of $\mathrm{Al}_{2} \mathrm{O}_{3}$ as a by-product. However, the corrosion resistance can be improved by the addition of non-wetting additives that react with mullite to form complex oxides that increase the alloy wetting resistance of the refractory surface [25-29].

More broadly, the corrosion resistance of mullite to some transition metals, such as $\mathrm{Ti}, \mathrm{V}, \mathrm{Fe}, \mathrm{Zn}$, and $\mathrm{Zr}$ is poor [12]. However, its corrosion resistance to $\mathrm{Ni}$ is good, which suggests the possibility of that mullite may be suitable for containment of Ni-based superalloys. There do not appear to be any studies of the corrosion resistance to Co-based superalloys.

Table 1 summarises the published literature on the production of mullite from fly ash. These data reveal that the foci of much of this work were the modification of the $\mathrm{Al}_{2} \mathrm{O}_{3}: \mathrm{SiO}_{2}$ ratio to shift it nearer to the stoichiometric $3: 2$ mullite ratio $\left(\sim 71.8 \mathrm{wt} \% \mathrm{Al}_{2} \mathrm{O}_{3}\right.$, $28.2 \mathrm{wt} \% \mathrm{SiO}_{2}$ ) and to produce porous mullite products. The production of porous bodies generally involves the use of $\mathrm{AlF}_{3}$, which tends to produce gaseous $\mathrm{SiF}_{4}$ upon reaction with the $\mathrm{SiO}_{2}$-rich glass present in these compositions, which removes the glass and creates porosity. More critically, heating was done for a maximal time of only $4 \mathrm{~h}$ in all of these studies (except for one where they formed whiskers at a relatively lower temperature) and none reported the long-term firing shrinkages. The combination of these factors represents the principal difference between these works and the present work, which reports the development of unique, dense, mullite microstructures revealed by firing shrinkage measurements and that are generated using high- $\mathrm{SiO}_{2}$ compositions and longterm heating. This novel composition and microstructure, which consists of percolated mullite, exhibits unparalleled high-temperature stability owing to the achievement of a continuous, direct-bonded, single-crystal network of mullite needles [30]. 
Table 1. Comprehensive summary of research on mullite production from fly ash.

\begin{tabular}{|c|c|c|c|c|c|}
\hline No. & Raw Materials & $\begin{array}{l}\text { Sintering Temperatures } \\
\left({ }^{\circ} \mathrm{C}\right) / \text { Time }\end{array}$ & $\begin{array}{l}\text { Microstructural Features and } \\
\text { Mullite Morphologies }\end{array}$ & Observations & Ref. \\
\hline 1 & Fly Ash/Bauxite & $1000^{\circ}-1600^{\circ} \mathrm{C} / 4 \mathrm{~h}$ & $\begin{array}{l}\text { - Porous microstructures at } \\
\text { - } \quad \text { Dewer temperatures } \\
\text { at } 1600^{\circ} \mathrm{C}\end{array}$ & $\begin{array}{l}\text { - At temperatures }<1300{ }^{\circ} \mathrm{C} \text {, cristobalite reacted with alumina } \\
\text { to form secondary mullite. } \\
\text { Above } 1400{ }^{\circ} \mathrm{C} \text {, mullite was the only crystalline phase in the } \\
\text { sintered samples. } \\
\text { - Samples sintered at } 1600^{\circ} \mathrm{C} \text { for } 4 \mathrm{~h} \text { had closed porosity of } \\
5.44 \% \text { and open porosity of } 0.62 \% \text {. }\end{array}$ & {$[31]$} \\
\hline 2 & $\begin{array}{l}\text { Fly Ash/Ammonium } \\
\text { Alum/Sodium } \\
\text { Dihydrogen } \\
\text { Phosphate }\end{array}$ & $1300^{\circ} \mathrm{C} / 10 \mathrm{~h}$ & $\begin{array}{l}\text { - Whiskers (aspect ratio of }>30 \text {; } \\
0.6-1.8 \mu \mathrm{m} \text { diameters) }\end{array}$ & $\begin{array}{l}\text { - Mullite whiskers were formed by reaction at } 1300{ }^{\circ} \mathrm{C} \text { for } 10 \mathrm{~h} \text {. } \\
\text { - Whiskers had a composition of } 47.11 \mathrm{~mol}^{\circ} \mathrm{SiO}_{2} \text { and } \\
52.89 \mathrm{~mol} \% \mathrm{Al}_{2} \mathrm{O}_{3} \text {. }\end{array}$ & [32] \\
\hline 3 & $\begin{array}{c}\text { Fly Ash/ } \\
\text { Bauxite } / \mathrm{V}_{2} \mathrm{O}_{5} / \mathrm{AlF}_{3}\end{array}$ & $1200^{\circ}-1500^{\circ} \mathrm{C} / 2.5 \mathrm{~h}$ & $\begin{array}{l}\text { Porous microstructures } \\
\text { (without additives) } \\
\text { Denser interlocked mullite } \\
\text { microstructures } \\
\text { (with additives) }\end{array}$ & $\begin{array}{l}\text { Porous microstructures consisted of rod-like mullite crystals } \\
\text { with no additives. } \\
\text { Addition of } 4 \mathrm{wt} \% \mathrm{AlF}_{3} \text { and } 3 \mathrm{wt} \% \mathrm{~V}_{2} \mathrm{O}_{5} \text { was optimal in } \\
\text { creating interlocked mullite microstructure with } 50 \% \text { open } \\
\text { porosity; secondary mullite formation was enhanced by } \\
\mathrm{V}_{2} \mathrm{O}_{5} \text { addition. } \\
\text { Interlocked mullite whiskers exhibited aspect ratios } \leq 18 \text {. }\end{array}$ & [33] \\
\hline 4 & $\begin{array}{c}\text { Fly } \\
\text { Ash/Bauxite } / \mathrm{TiO}_{2}\end{array}$ & $1300^{\circ}-1500^{\circ} \mathrm{C} / 2 \mathrm{~h}$ & $\begin{array}{l}\text { Porous microstructures with } \\
\text { gradual densification with } \\
\text { increasing } \mathrm{TiO}_{2} \text { amounts }\end{array}$ & $\begin{array}{l}\text { - Samples sintered at } 1450{ }^{\circ} \mathrm{C} \text { for } 2 \mathrm{~h} \text { exhibited the lowest firing } \\
\text { shrinkage and bulk density and the greatest open porosities } \\
\text { at } \mathrm{TiO}_{2} \text { contents of } 0-6 \mathrm{wt} \% \text {. }\end{array}$ & {$[34]$} \\
\hline 5 & $\begin{array}{c}\text { Fly Ash/ } \\
\mathrm{Al}(\mathrm{OH})_{3} / \mathrm{Al}_{2} \mathrm{O}_{3} / \mathrm{AlF}_{3}\end{array}$ & $1400^{\circ}-1600^{\circ} \mathrm{C} / 4 \mathrm{~h}$ & $\begin{array}{l}\text { - } \quad \text { Reinforced needle-like whiskers } \\
\text { - } \quad \mathrm{Al}(\mathrm{OH})_{3} \text { addition } \\
\text { - } \quad \mathrm{Short} \text { whiskers with } \\
\mathrm{Al}_{2} \mathrm{O}_{3} \text { additions } \\
\text { - } \quad \mathrm{Granular} \text { particles with } \\
\mathrm{AlF}_{3} \text { additions }\end{array}$ & $\begin{array}{l}\text { - Interlocked needle-like mullite whiskers were formed. } \\
\text { This microstructure enhanced the bend strength of porous } \\
\text { mullite, with a value of } 100 \mathrm{MPa} \text { at an apparent porosity of } \\
\sim 55 \% \text { after sintering at } 1550^{\circ} \mathrm{C} \text {. }\end{array}$ & [35] \\
\hline
\end{tabular}


Table 1. Cont.

\begin{tabular}{|c|c|c|c|c|c|}
\hline No. & Raw Materials & $\begin{array}{l}\text { Sintering Temperatures } \\
\left({ }^{\circ} \mathrm{C}\right) / \text { Time }\end{array}$ & $\begin{array}{l}\text { Microstructural Features and } \\
\text { Mullite Morphologies }\end{array}$ & Observations & Ref. \\
\hline 6 & $\begin{array}{c}\text { Fly Ash/ } \\
\text { Bauxite/ } \mathrm{V}_{2} \mathrm{O}_{5}\end{array}$ & $1100^{\circ}-1500^{\circ} \mathrm{C} / 4 \mathrm{~h}$ & $\begin{array}{l}\text { Puncheon-shaped (aspect } \\
\text { ratio of } 6 \text { ) to equiaxed } \\
\text { mullite crystals }\end{array}$ & $\begin{array}{l}\text { - Increasing } \mathrm{V}_{2} \mathrm{O}_{5} \text { content at } 1500{ }^{\circ} \mathrm{C} \text { increased the apparent } \\
\text { porosity and water absorption. } \\
\text { Mullite morphology changed from small puncheons to large } \\
\text { cuboids with increasing temperature. }\end{array}$ & [36] \\
\hline 7 & $\begin{array}{l}\text { Fly Ash Hollow } \\
\text { Spheres (FAHSs) }\end{array}$ & $1250^{\circ}-1400^{\circ} \mathrm{C} / 3 \mathrm{~h}$ & $\begin{array}{l}\text { - Individual hollow spheres } \\
\text { bonded to form foam } \\
\text { Elongation of mullite grains } \\
\text { on FAHS shells with } \\
\text { increasing temperature }\end{array}$ & $\begin{array}{l}\text { - Fracture mechanism of FAHS ceramic foams changed from } \\
\text { intergranular to transgranular when temperature increased } \\
\text { from } 1250^{\circ} \text { to } 1400^{\circ} \mathrm{C} \text {. } \\
\text { Higher compressive strengths of } \sim 6.7 \mathrm{MPa} \text { observed after } \\
\text { sintering at } 1400^{\circ} \mathrm{C} \text { were attributed to the formation of } \\
\text { strong necking between FAHS during sintering. }\end{array}$ & [37] \\
\hline 8 & $\begin{array}{c}\text { Fly } \\
\text { Ash/Bauxite/MgO }\end{array}$ & $1300^{\circ}-1500^{\circ} \mathrm{C} / 2 \mathrm{~h}$ & $\begin{array}{l}\text { - } \quad \text { Needles in glassy phase } \\
\text { Needle interlocking } \\
\text { at } 1500{ }^{\circ} \mathrm{C}\end{array}$ & $\begin{array}{l}\text { - } \mathrm{MgO} \text { addition promoted densification and increased the } \\
\text { strengths after sintering at }>1400{ }^{\circ} \mathrm{C} \text {. } \\
\mathrm{MgO} \text { additions resulted in the formation of low-expansion } \\
\alpha \text {-cordierite at } 1300{ }^{\circ} \mathrm{C} \text { while corundum and spinel were } \\
\text { formed at } 1400{ }^{\circ} \mathrm{C} \text {. }\end{array}$ & [38] \\
\hline 9 & Desilicified Fly Ash & $1300^{\circ}-1600^{\circ} \mathrm{C} / 4 \mathrm{~h}$ & $\begin{array}{l}\text { Lath-like mullite crystals that } \\
\text { interlocked to form a } \\
\text { framework structure filled } \\
\text { with glassy phase at } 1600^{\circ} \mathrm{C}\end{array}$ & $\begin{array}{l}\text { - Desilicified fly ash exhibited superior physical and } \\
\text { mechanical properties compared to samples from fly ash. } \\
\text { Lath-like and needle-like mullite crystals formed in high-iron } \\
\text { and low-iron samples, respectively. } \\
\text { Lath-like structures increased the flexural strengths } \\
\text { compared to those with needle-like structures. }\end{array}$ & [39] \\
\hline 10 & $\begin{array}{l}\text { High-Aluminium } \\
\text { Fly Ash }\end{array}$ & $1200^{\circ}-1600^{\circ} \mathrm{C} / 2 \mathrm{~h}$ & $\begin{array}{l}\text { Network structure of } \\
\text { anisotropic grains } \\
(10 \mu \mathrm{m} \text { lengths }) \text { with minimal } \\
\text { glass phase }\end{array}$ & $\begin{array}{l}\text { - Alkali and acid treatment were done in order to increase the } \\
\mathrm{Al}_{2} \mathrm{O}_{3} \text { content to } \sim 65 \mathrm{wt} \% \text { while removing impurities. } \\
\text { Samples sintered at } 1600{ }^{\circ} \mathrm{C} \text { exhibited apparent porosity, bulk } \\
\text { density, and compressive strength of } 1.2 \%, 2780 \mathrm{~kg} \cdot \mathrm{m}^{-3} \text {, and } \\
169 \mathrm{MPa} \text {, respectively. }\end{array}$ & [40] \\
\hline
\end{tabular}


Table 1. Cont.

\begin{tabular}{|c|c|c|c|c|c|}
\hline No. & Raw Materials & $\begin{array}{l}\text { Sintering Temperatures } \\
\left({ }^{\circ} \mathrm{C}\right) / \text { Time }\end{array}$ & $\begin{array}{l}\text { Microstructural Features and } \\
\text { Mullite Morphologies }\end{array}$ & Observations & Ref. \\
\hline 11 & $\begin{array}{l}\text { Fly Ash/ } \\
\text { Bauxite/Potash } \\
\text { Feldspar/SiC } / \mathrm{V}_{2} \mathrm{O}_{5}\end{array}$ & $1450^{\circ}-1550^{\circ} \mathrm{C} / 2 \mathrm{~h}$ & $\begin{array}{l}\text { Prismatic mullite crystals } \\
\text { forming interlocking } \\
\text { microstructure }\end{array}$ & $\begin{array}{l}\text { - With } 0 \text { to } 15 \mathrm{wt} \% \mathrm{SiC} \text { addition, prismatic mullite crystals } \\
\text { increased in number, which increased the thermal } \\
\text { conductivity and strength. } \\
\text { With } 12 \mathrm{wt} \% \text { potash feldspar addition, the compressive } \\
\text { strength increased and the thermal conductivity reduced } \\
\text { relative to the preceding; samples survived seven thermal } \\
\text { shock cycles. }\end{array}$ & [41] \\
\hline 12 & Fly Ash $/ \mathrm{SiC} / \mathrm{MoO}_{3}$ & $\begin{array}{l}850^{\circ} \mathrm{C} / 2 \mathrm{~h} \\
1000^{\circ} \mathrm{C} / 1 \mathrm{~h}\end{array}$ & $\begin{array}{l}\text { Increased rod-shaped mullite } \\
\text { crystals (from } 0.8 \mu \mathrm{m} \text { to } \\
10 \mu \mathrm{m} \text { ) at higher temperature }\end{array}$ & $\begin{array}{l}\text { - } 5 \mathrm{wt} \% \mathrm{MoO}_{3} \text { addition resulted in achievement of the optimal } \\
\text { strength of the mullite-bonded } \mathrm{SiC} \text {. }\end{array}$ & {$[42]$} \\
\hline 13 & Fly Ash/Boehmite Sol & $900^{\circ}-1300^{\circ} \mathrm{C} / 2 \mathrm{~h}$ & $\begin{array}{l}\text { Prismatic crystallites } \\
(<0.3 \mu \mathrm{m}) \text { from } 4 \mathrm{wt} \% \\
\text { boehmite addition } \\
\text { Prismatic crystallites } \\
(0.3-1.0 \mu \mathrm{m}) \text { from } 12 \% \\
\text { boehmite addition }\end{array}$ & $\begin{array}{l}\text { - Optimal mullite content }(63.8 \%) \text { was achieved with } 12 \mathrm{wt} \% \\
\text { boehmite with sintering at } 1200{ }^{\circ} \mathrm{C} \text {. }\end{array}$ & [43] \\
\hline 14 & $\begin{array}{c}\text { Fly } \\
\mathrm{Ash} / \mathrm{Al}(\mathrm{OH})_{3} / \mathrm{MoO}_{3}\end{array}$ & $1100^{\circ}-1500^{\circ} \mathrm{C} / 2 \mathrm{~h}$ & $\begin{array}{l}\text { Increased liquid phase } \\
\text { formation and larger } \\
\text { aspect ratios of interlocked } \\
\text { mullite whiskers from } \\
\text { increasing } \mathrm{MoO}_{3}\end{array}$ & $\begin{array}{l}\text { - With } 0 \text { to } 20 \mathrm{wt} \% \mathrm{MoO}_{3} \text { addition, open porosity significantly } \\
\text { increased from } 42 \% \text { to } 58 \% \text { with sintering at } 1300^{\circ} \mathrm{C} \text {. } \\
20 \mathrm{wt} \% \mathrm{MoO}_{3} \text { addition increased the flexural strength at } \\
\text { higher sintering temperatures. }\end{array}$ & [44] \\
\hline 15 & $\begin{array}{l}\text { High-Alumina Fly } \\
\text { Ash/HF/NaOH }\end{array}$ & $1100^{\circ}-1400^{\circ} \mathrm{C} / 2 \mathrm{~h}$ & $\begin{array}{l}\text { - Cubic puncheon-shaped } \\
\text { mullite crystals }\end{array}$ & $\begin{array}{l}\text { Alkali-activation treatment adjusted the chemical } \\
\text { composition to that of stoichiometric mullite. } \\
\text { 100\% fly-ash-based ceramic tiles sintered at } 1300^{\circ} \mathrm{C} \text { exhibited } \\
\text { optimal postsintered densification. }\end{array}$ & {$[45]$} \\
\hline
\end{tabular}


Table 1. Cont.

\begin{tabular}{|c|c|c|c|c|c|}
\hline No. & Raw Materials & $\begin{array}{l}\text { Sintering Temperatures } \\
\left({ }^{\circ} \mathrm{C}\right) / \text { Time }\end{array}$ & $\begin{array}{l}\text { Microstructural Features and } \\
\text { Mullite Morphologies }\end{array}$ & Observations & Ref. \\
\hline 16 & $\begin{array}{c}\text { Fly } \\
\text { Ash/Alumina/ } \mathrm{AlF}_{3}\end{array}$ & $1000^{\circ}-1400^{\circ} \mathrm{C} / 2 \mathrm{~h}$ & $\begin{array}{l}\text { - Increased distribution density } \\
\text { and aspect ratio of rod-like } \\
\text { mullite crystals with } \\
\text { increasing } \mathrm{AlF}_{3} \text { content } \\
\text { Transition from rod- to } \\
\text { whisker-shaped mullite with } \\
\text { increasing temperature }\end{array}$ & $\begin{array}{l}\text { - Fly ash with } 6 \mathrm{wt} \% \mathrm{AlF}_{3} \text { sintered at } 1200{ }^{\circ} \mathrm{C} \text { exhibited lower } \\
\text { apparent porosity and higher flexural strength than that of } \\
\text { samples prepared from } \mathrm{HCl} \text {-pickled fly ash. }\end{array}$ & [46] \\
\hline 17 & $\begin{array}{l}\text { Fly Ash } / \mathrm{Na}_{2} \mathrm{SO}_{4} / \\
\text { Aluminium } \\
\text { Sulfate/HF }\end{array}$ & $1000^{\circ} \mathrm{C} / 3 \mathrm{~h}$ & $\begin{array}{l}\text { - Whiskers (aspect ratio }>25 \text {, } \\
0.06-0.3 \mu \mathrm{m} \text { widths) }\end{array}$ & $\begin{array}{l}\text { - Mullite whiskers were prepared by varying the } \\
\text { additive ratios }\end{array}$ & [47] \\
\hline 18 & $\begin{array}{l}\text { Fly Ash/Bauxite/ } \\
\text { Kaolin/Potash } \\
\text { Feldspar/ } \\
\text { Talc/BaCO } 3 \text { /Pyrolusite }\end{array}$ & $1390^{\circ} \mathrm{C} / 2 \mathrm{~h}$ & $\begin{array}{l}\text { - Thin layer of interwoven } \\
\text { anisotropic mullite crystals of } \\
\text { well controlled crystal size }\end{array}$ & $\begin{array}{l}\text { - Network of mullite-whisker-reinforced ceramics were } \\
\text { prepared by sintering. } \\
\text { Maximal strength and minimal bulk density resulted from } \\
\text { composition containing } 2 \mathrm{wt} \% \text { potash feldspar, } 2 \mathrm{wt} \% \text { talc, } \\
1 \mathrm{wt} \% \mathrm{BaCO}_{3} \text {, and } 6 \mathrm{wt} \% \text { pyrolusite. }\end{array}$ & [48] \\
\hline 19 & $\begin{array}{l}\text { High-Aluminium Fly } \\
\text { Ash/NaOH }\end{array}$ & $1400{ }^{\circ} \mathrm{C} / 100 \mathrm{~min}$ & $\begin{array}{l}\text { Microstructures containing } \\
\text { small numbers of } 15-25 \mu \mathrm{m} \\
\text { mullite/corundum particles } \\
\text { and large numbers of } \\
5-15 \mu \mathrm{m} \text { mullite/corundum } \\
\text { particles } \\
\text { Needle-like recrystallised } \\
\text { mullite between } \\
\text { mullite/corundum particles }\end{array}$ & $\begin{array}{l}\text { - Sintering at } 1400{ }^{\circ} \mathrm{C} \text { resulted in the maximal mechanical } \\
\text { properties and minimal apparent porosity. }\end{array}$ & [49] \\
\hline 20 & $\begin{array}{l}\text { Fly Ash Cenospheres } \\
\text { (FACs)/Kaolin }\end{array}$ & $900^{\circ}-1300^{\circ} \mathrm{C} / 3 \mathrm{~h}$ & $\begin{array}{l}\text { - Homogenous microstructure } \\
\text { with FACs in continuous } \\
\text { mullite matrix }\end{array}$ & $\begin{array}{l}\text { - FAC / mullite composites exhibited low bulk densities } \\
\left(650-1380 \mathrm{~kg} \cdot \mathrm{m}^{-3}\right) \text {, high apparent porosities }(23.36-70.32 \%) \text {, } \\
\text { low thermal conductivities }\left(0.14-0.45 \mathrm{~W} \cdot \mathrm{m}^{-1} \mathrm{~K}^{-1}\right) \text {, and } \\
\text { relatively high compressive strengths }(1.56-32.99 \mathrm{MPa}) \text {. } \\
50 \mathrm{wt} \% \mathrm{FAC}+50 \mathrm{wt} \% \text { kaolin sintered at } 1200^{\circ} \mathrm{C} \text { exhibited a } \\
\text { firing shrinkage of } 9.45 \% \text {. }\end{array}$ & [50] \\
\hline
\end{tabular}


Table 1. Cont.

\begin{tabular}{|c|c|c|c|c|c|}
\hline No. & Raw Materials & $\begin{array}{l}\text { Sintering Temperatures } \\
\left({ }^{\circ} \mathrm{C}\right) / \text { Time }\end{array}$ & $\begin{array}{l}\text { Microstructural Features and } \\
\text { Mullite Morphologies }\end{array}$ & Observations & Ref. \\
\hline 21 & $\begin{array}{l}\text { High-Aluminium Fly } \\
\text { Ash } / \mathrm{HCl}\end{array}$ & $1300^{\circ}-1500^{\circ} \mathrm{C} / 2-4 \mathrm{~h}$ & $\begin{array}{l}\text { Sintered mullite needle length } \\
\text { from as-received fly ash } \\
\text { double that from beneficiated } \\
\text { fly ash } \\
\text { Increasing mullite needle } \\
\text { length with increasing } \\
\text { sintering temperature }\end{array}$ & $\begin{array}{l}\text { Highest compressive strengths achieved at optimal sintering } \\
\text { temperatures of } 1400^{\circ} \mathrm{C} \text { for the as-received fly ash and } \\
1500{ }^{\circ} \mathrm{C} \text { for the beneficiated fly ash. }\end{array}$ & {$[51]$} \\
\hline 22 & $\begin{array}{l}\text { Fly Ash + Alumina + } \\
\text { Calcite }\end{array}$ & $\begin{array}{l}1400^{\circ}-1800^{\circ} \mathrm{C} / \\
\text { Time not specified }\end{array}$ & $\begin{array}{l}-0.1-2.0 \mu \mathrm{m} \text { long } \\
\text { rod-like mullite }\end{array}$ & $\begin{array}{l}\text { - Sintering at } 1700^{\circ} \mathrm{C} \text { resulted in mullite formation but at } \\
1800{ }^{\circ} \mathrm{C} \text { resulted in the formation of equal amounts of mullite } \\
\text { and corundum plus a minor amount of anorthite. }\end{array}$ & {$[52]$} \\
\hline 23 & $\begin{array}{l}\text { Fly Ash/Aluminium } \\
\text { Dross / HCl }\end{array}$ & $1200^{\circ}, 1500^{\circ} \mathrm{C} / 4 \mathrm{~h}$ & $\begin{array}{l}\text { Acicular mullite crystals } \\
\text { embedded in aluminosilicate } \\
\text { glass matrix }\end{array}$ & $\begin{array}{l}\text { Following beneficiation in } 2 \mathrm{M} \mathrm{HCl} \text { for } 1 \mathrm{~h} \text {, the mullite-based } \\
\text { product was well crystallised and exhibited low thermal } \\
\text { expansion and smooth expansion curve. }\end{array}$ & [53] \\
\hline 24 & Fly Ash/Bauxite & $\begin{array}{l}1300^{\circ}-1500^{\circ} \mathrm{C} / \text { Time } \\
\text { not specified }\end{array}$ & $\begin{array}{l}\text { Lath-like mullite crystals, } \\
\text { glassy phase, and uniformly } \\
\text { distributed pores }\end{array}$ & $\begin{array}{l}\text { - Sample fired at } 1450{ }^{\circ} \mathrm{C} \text { resulted in a bulk density of } \\
2100 \mathrm{~kg} \cdot \mathrm{m}^{-3} \text { and apparent porosity of } \sim 32 \% \text {. }\end{array}$ & [54] \\
\hline 25 & $\begin{array}{l}\text { Fly } \\
\mathrm{Ash} / \mathrm{Al}_{2} \mathrm{O}_{3} / \text { Starch } \\
\text { Filler }\end{array}$ & $1600{ }^{\circ} \mathrm{C} / 4 \mathrm{~h}$ & $\begin{array}{l}\text { - Microstructure of interwoven } \\
\text { mullite and glassy phase }\end{array}$ & $\begin{array}{l}\text { - Major mullite and minor corundum increased with increasing } \\
\mathrm{Al}_{2} \mathrm{O}_{3} \text { content. } \\
\text { Pure fly ash resulted in interwoven mullite needles and high } \\
\text { volume of glass. } \\
\text { Al/Si molar ratio of } 2.40 \text { resulted in highest linear firing } \\
\text { shrinkages }(\sim 29 \%) \text { and compressive strengths }(\sim 2.3 \mathrm{MPa}) \\
\text { with } \sim 77 \% \text { apparent porosity from starch filler. }\end{array}$ & [55] \\
\hline
\end{tabular}




\section{Percolated Mullite}

Highly dense 3:2 mullite ( $<2 \%$ apparent porosity) with microstructures completely percolated throughout the entire sample volumes were fabricated using either fly ash alone or fly ash mixed with an oxide addition at a low concentration $[30,56]$. These intergrown microstructures developed through mullite nucleation (small equiaxed precipitated grains of primary mullite) and growth (elongated grains of secondary mullite from primary mullite and pre-existing mullite needles). Although percolation can be achieved using fly ash alone, the kinetics of percolation can be enhanced through the addition of different additive types and amounts. A key but counter-intuitive feature of the generation of percolated mullite is the presence of excess $\mathrm{SiO}_{2}$-rich glass. This glass acts as both a medium for the diffusion of ions for mullite growth at high temperatures but also provides a deformable medium that is compliant with the axial and diametral growth of the mullite needles. Once these needles have grown to sufficient lengths, they form direct-bonded intergrowths that completely exclude any glass between the grains [57], thereby forming a non-shrinking single-crystal network that exhibits the intrinsic properties of pure mullite on a full volumetric basis [56,57]. Critically, this percolated microstructure isolates any residual glass within the triple points, effectively forming a 0-3 composite [58,59] whose thermomechanical properties, including high-temperature strength and creep [56], are unaffected by the glass. The thermal expansion would be expected to be increased by the glass but this effect would be mitigated by resistance from the rigid, continuous, mullite skeleton.

The percolated mullite was developed and patented over the period 2012-2016 from a range of Class F fly ashes from Australia and China; typical compositions for these are shown in Table 2. These fly ashes contain glass as the major component, with minor crystalline phases of mullite and $\alpha$-quartz plus trace amounts of magnetite and $\alpha$-hematite.

Table 2. Typical oxide contents of fly ashes and stoichiometric mullite (wt\%).

\begin{tabular}{ccccccccccc}
\hline Sample & $\mathbf{S i O}_{\mathbf{2}}$ & $\mathbf{A l}_{\mathbf{2}} \mathbf{O}_{\mathbf{3}}$ & $\mathbf{F e}_{\mathbf{2}} \mathbf{O}_{\mathbf{3}}$ & $\mathbf{N a}_{\mathbf{2}} \mathbf{O}$ & $\mathbf{K}_{\mathbf{2}} \mathbf{O}$ & $\mathbf{C a O}$ & $\mathbf{M g O}$ & $\mathrm{TiO}_{\mathbf{2}}$ & $\mathbf{L O I}$ & $\mathbf{O}$ ther \\
\hline Fly Ash 1 & 72.2 & 22.6 & 1.1 & 0.1 & 0.5 & 0.0 & 0.2 & 1.3 & 2.1 & 0.0 \\
Fly Ash 2 & 33.8 & 31.2 & 2.5 & 0.1 & 0.4 & 1.6 & 0.4 & 1.3 & 25.0 & 3.7 \\
Fly Ash 3 & 68.1 & 23.6 & 1.3 & 0.2 & 2.2 & 0.7 & 0.3 & 0.9 & 2.4 & 0.3 \\
General Range & $33-75$ & $18-36$ & $0.8-6.0$ & $0.0-0.3$ & $0.4-2.6$ & $0.1-4.0$ & $0.2-1.0$ & $0.4-1.8$ & $0.5-25$ & $0.5-4.0$ \\
Mullite & 28.2 & 71.8 & 0.0 & 0.0 & 0.0 & 0.0 & 0.0 & 0.0 & 0.0 & 0.0 \\
\hline
\end{tabular}

The pure fly ash compositions were uniaxially pressed into cylindrical pellets $(15 \mathrm{~mm}$ diameter $\times 5 \mathrm{~mm}$ height), heated to $1500{ }^{\circ} \mathrm{C}$ (heating rate $120{ }^{\circ} \mathrm{C} / \mathrm{h}$ ), and soaked for 1-96 h. Figure 2 shows the changes in mullite needle dimensions (lengths and width) with increasing heat treatment time. All of the microstructures were etched with HF and then characterised using scanning electron microscopy (SEM). The samples were not carbon coated since this could have impacted on the imaging resolution between the individual needles. However, the result of this was some charging of the samples, as indicated by the lighter coloured regions in the images. It can be seen that the well-formed but discrete mullite needles formed at only $1 \mathrm{~h}$ but these developed into a continuous interconnected network by $4 \mathrm{~h}$. At the longer time points, the main difference was needle coarsening, increasing aspect ratio, and some exaggerated grain growth.

Importantly, the diametral firing shrinkages were determined at each sintering time. Figure 3 shows the diametral firing shrinkage trends of the pure fly ashes $(1,2$, and 3$)$ and fly ash 3 with $\alpha-\mathrm{Al}_{2} \mathrm{O}_{3}$ added in order to shift the overall composition toward that of mullite $\left(\sim 46 \mathrm{wt} \% \alpha-\mathrm{Al}_{2} \mathrm{O}_{3}\right)$. These data clarify that that percolation, indicated by the cessation of further volumetric firing shrinkage, can be achieved at a soak time of only $2 \mathrm{~h}$ at $1500^{\circ} \mathrm{C}$. 


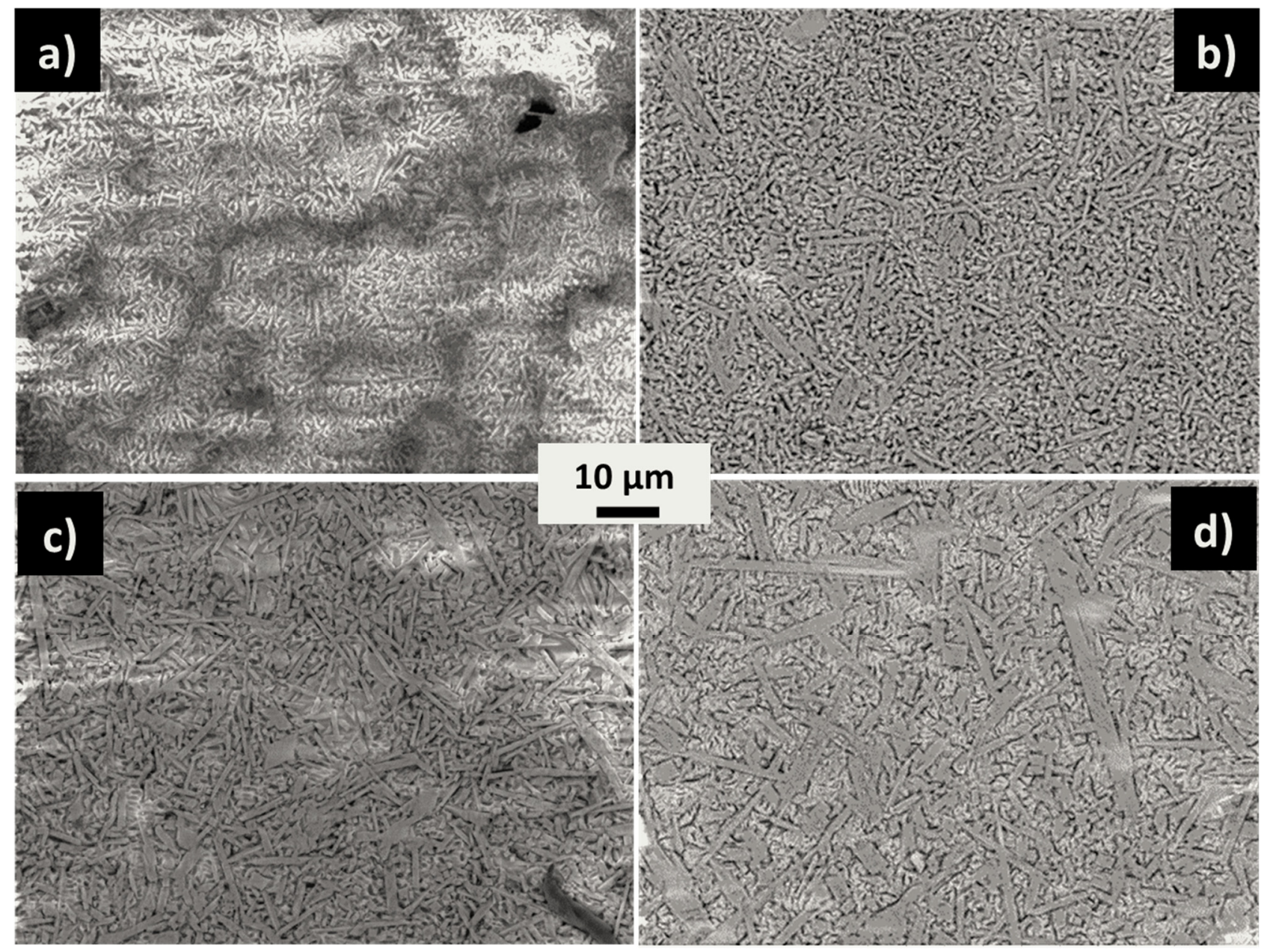

Figure 2. Microstructural variations in pure fly ash 2 samples sintered at $1500{ }^{\circ} \mathrm{C}$ for different times (a) $1 \mathrm{~h},(\mathbf{b}) 4 \mathrm{~h},(\mathbf{c}) 24 \mathrm{~h}$, and (d) $96 \mathrm{~h}$.

a)

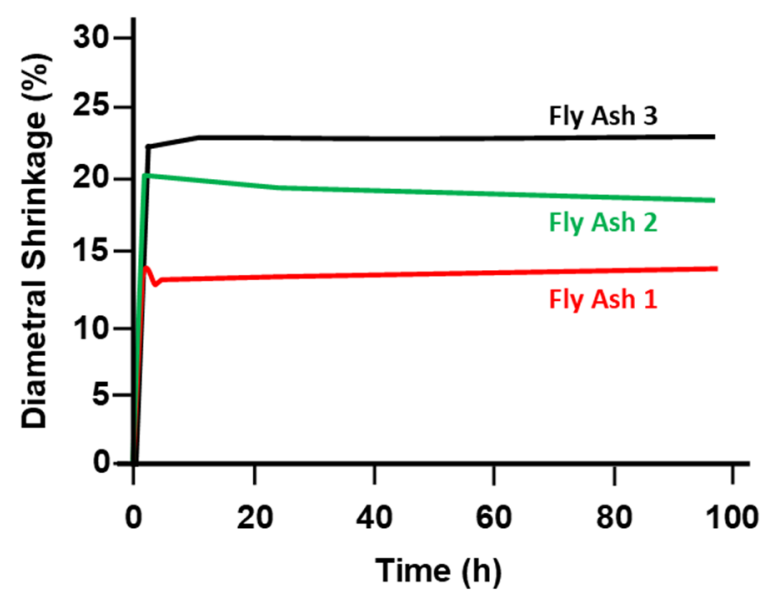

b)

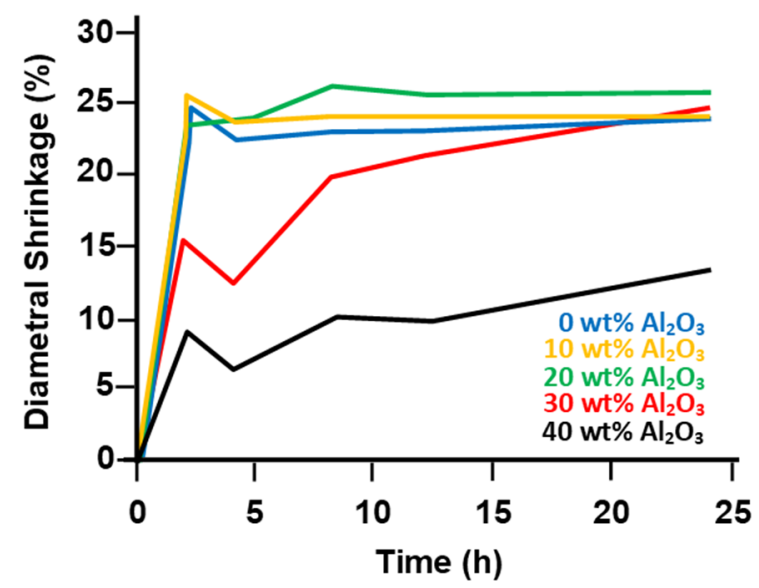

Figure 3. (a) Diametral firing shrinkages of pure fly ashes $(1,2$, and 3$)$ after sintering at $1500{ }^{\circ} \mathrm{C}$ for $\leq 96 \mathrm{~h}$, (b) Diametral firing shrinkages of fly ash 3 with alumina additions after sintering at $1500{ }^{\circ} \mathrm{C}$ for $\leq 24 \mathrm{~h}$ (Adapted from [30]). 
It is somewhat surprising that, despite the differences between the fly ashes in terms of amount and type of glass and the amount of mullite, percolation could be achieved consistently at soak times of $\sim 2-4 \mathrm{~h}$. The major differences were manifested in the extents of firing shrinkage for pure fly ashes and in the kinetics of firing shrinkage for the fly ashes with $\alpha-\mathrm{Al}_{2} \mathrm{O}_{3}$ added. The principal driving force for these differences is shown in Table 2, which reveals significantly different (1) $\mathrm{Al}_{2} \mathrm{O}_{3} / \mathrm{SiO}_{2} \mathrm{wt} \%$ ratios, (2) amounts of fluxing impurities (e.g., $\mathrm{Na}_{2} \mathrm{O}, \mathrm{K}_{2} \mathrm{O}, \mathrm{CaO}$, and $\mathrm{Fe}_{2} \mathrm{O}_{3}$ ), (3) resultant glass viscosity, and (4) amounts of unburnt carbon (shown by the loss on ignition, LOI). The first of these variables would affect both the glass viscosity but also the ultimate mullite content. The second of these would affect the glass viscosity but also the tendency to favour the formation of equiaxed or needle-like mullite [12]. The last of these would affect the firing shrinkage upon oxidative gas emission.

When comparing the firing shrinkage behaviour of samples fabricated using only fly ashes with those fabricated using mixtures of fly ash and calcined alumina, it is clear that the addition of $\alpha-\mathrm{Al}_{2} \mathrm{O}_{3}$ has an insignificant effect at low additions $(<20 \mathrm{wt} \%)$ and a retarding effect at high additions $(>20 \mathrm{wt} \%$ ) on the achievement of percolation. This clearly reflects the role of $\mathrm{Al}_{2} \mathrm{O}_{3}$ in increasing the glass viscosity, as suggested in Figure $1 \mathrm{a}$. When $\alpha-\mathrm{Al}_{2} \mathrm{O}_{3}$ is added, the diametral firing shrinkage shows a fluctuation (viz., expansion) at $4 \mathrm{~h}$, which was observed in fly ash 1 . This expansion is interpreted in terms of the sequence prior to percolation described in Table 3.

Table 3. Interpretation of volumetric shrinkage prior to percolation exhibited in Figure 3.

\begin{tabular}{cccl}
\hline Time & Shrinkage & Glass Viscosity & \multicolumn{1}{c}{ Dominant Effect } \\
\hline$<2 \mathrm{~h}$ & $\begin{array}{c}\text { Significant } \\
\text { contraction }\end{array}$ & High & $\begin{array}{l}\text { Volumetric shrinkage from softening of solid-like glass with little effect } \\
\text { of dispersed mullite needles in deformable matrix }\end{array}$ \\
\hline $2-4 \mathrm{~h}$ & $\begin{array}{c}\text { Minor } \\
\text { expansion }\end{array}$ & High & $\begin{array}{l}\text { Volumetric expansion from thermal expansion of solid-like glass and } \\
\text { semi-percolated mullite needle skeleton }\end{array}$ \\
\hline$>4 \mathrm{~h}$ & $\begin{array}{c}\text { Continued } \\
\text { contraction }\end{array}$ & Decreasing & $\begin{array}{l}\text { Gradual volumetric shrinkage from resultant progressively decreasing } \\
\text { glass viscosity, and increasingly liquid-like behaviour }\end{array}$ \\
\hline
\end{tabular}

It also is possible that the expansion derived from gas exudation upon the achievement of sufficient reduction in glass viscosity and resultant microstructural disruption but these are not consistent with the LOI levels shown in Table 2.

The preceding considerations clarify the reasons for the failure of previous studies to report volumetric percolation. That is, in addition to the use of soak times generally insufficient to achieve percolation and the non-measurement of firing shrinkages, the glasses at issue were of high viscosities such that the necessary diffusion and compliance for mullite percolation were not established because (1) the studies of pure fly ash involved high- $\mathrm{Al}_{2} \mathrm{O}_{3}$ types and (2) the studies of fly ash with $\mathrm{Al}_{2} \mathrm{O}_{3}$ added were designed to equate to the mullite composition.

Image analysis of the SEM images was conducted to quantify the microstructural changes occurring during heat treatment as a function of soak time. For each SEM image, 500-1000 grains (depending on time) of mullite were measured in order to provide statistically significant quantification of the needle lengths in terms of the values D10, D50, average, and D90, as shown in Figure 4a. The data in Figure $4 \mathrm{~b}$ show that the needle lengths and areal coverage of the microstructure increased significantly with increasing sintering time up to $24 \mathrm{~h}$, with the most of the significant alterations occurring within $\sim 4 \mathrm{~h}$. Soaking for longer times reduced the number of mullite needles owing to grain growth, terminating in areal coverage of $\sim 96 \%$ and a maximal needle length (for fly ash 2 ) of $\sim 15 \mu \mathrm{m}$. 
a)

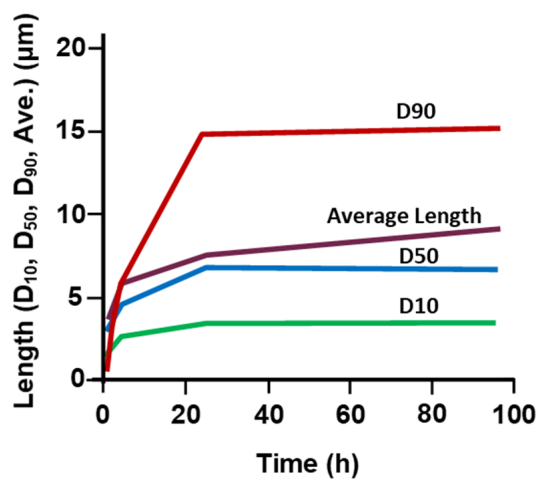

b)

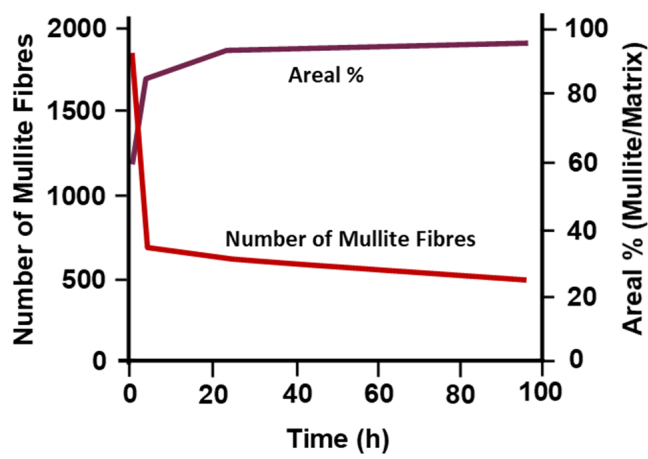

Figure 4. Fly ash 2: (a) changes to needle dimensions and their proportion with increasing sintering time at $1500{ }^{\circ} \mathrm{C},(\mathbf{b})$ Changes to needle numbers and areal coverage with increasing sintering time at $1500{ }^{\circ} \mathrm{C}$ (Adapted from [30]).

The kinetics to achieve percolation also can be manipulated through the addition of oxides that modify the glass viscosity. Figure 5 summarises the impacts of three additives: $\mathrm{MgO}(1 \mathrm{wt} \%), \mathrm{CaO}(0.5 \mathrm{wt} \%)$, and $\mathrm{Fe}_{2} \mathrm{O}_{3}(2 \mathrm{wt} \%)$. Comparison of Figures $3 \mathrm{~b}$ and 5 shows the general enhancement of the rate of percolation from the oxide additions. These data also reveal that $\alpha-\mathrm{Fe}_{2} \mathrm{O}_{3}$ was more effective than $\mathrm{MgO}$ or $\mathrm{CaO}$, which reflects a combination of the solidus temperatures of the respective systems and the amounts of the additives:

$\begin{array}{llll}\mathrm{FeO}-\mathrm{Al}_{2} \mathrm{O}_{3}-\mathrm{SiO}_{2} & 1083{ }^{\circ} \mathrm{C} & (1.8 \mathrm{wt} \% \text { addition }) & {[60]} \\ \mathrm{Fe}_{2} \mathrm{O}_{3}-\mathrm{Al}_{2} \mathrm{O}_{3}-\mathrm{SiO}_{2} & 1382{ }^{\circ} \mathrm{C} & (2.0 \mathrm{wt} \% \text { addition }) & {[61]} \\ \mathrm{MgO}_{\mathrm{Agl}} \mathrm{O}_{3}-\mathrm{SiO}_{2} & 13555^{\circ} \mathrm{C} & (1.0 \mathrm{wt} \% \text { addition }) & {[60]} \\ \mathrm{CaO}-\mathrm{Al}_{2} \mathrm{O}_{3}-\mathrm{SiO}_{2} & 1170{ }^{\circ} \mathrm{C} & (0.5 \mathrm{wt} \% \text { addition }) & {[60]}\end{array}$
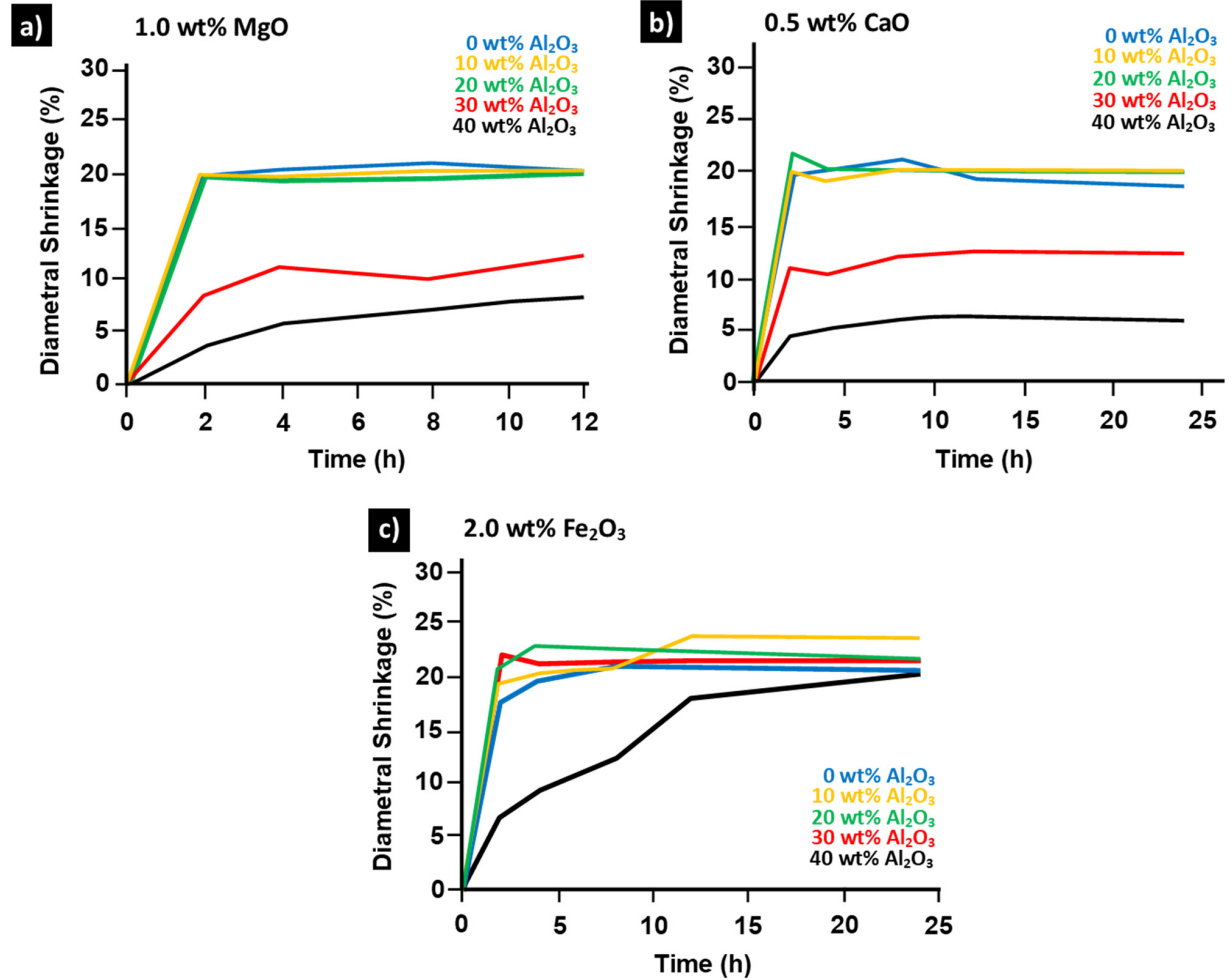

Figure 5. Diametral firing shrinkages of fly ash $3+$ alumina compositions at $1500{ }^{\circ} \mathrm{C}$ for extended times with the addition of (a) $1 \mathrm{wt} \% \mathrm{MgO}$, (b) $0.5 \mathrm{wt} \% \mathrm{CaO}$, and (c) $2 \mathrm{wt} \% \mathrm{Fe}_{2} \mathrm{O}_{3}$ (Adapted from [30]). 
The data for the equivalent $\mathrm{FeO}$ from $\alpha-\mathrm{Fe}_{2} \mathrm{O}_{3}$ are included because reduction of the latter to the former is a common occurrence during the heating in air of thick ceramics owing to oxygen starvation of the interior of the compact, as is evidenced frequently from the black coring of Fe-rich bricks [62]. The additions in the present work represent maximal addition levels as higher amounts resulted in excessive vitrification, loss of sample shape, and prevention of mullite percolation.

Figures $3-5$ show that percolation can be achieved in $\sim 2-4 \mathrm{~h}$ for fly ashes both without and with oxide addition, resulting in a $0-3[58,59]$ glass-mullite composite consisting of a dense, continuous, single-crystal, mullite skeleton with glass isolated in the triple points. These data also show that there is only insignificant microstructural alteration after heating at $1500{ }^{\circ} \mathrm{C}$ for $\sim 24 \mathrm{~h}$, so the grains undergo little additional grain growth. These data also show that the conventional logic of addition of $\mathrm{Al}_{2} \mathrm{O}_{3}$ to achieve a net mullite composition prevents the detection of percolation owing to high glass viscosity, insufficient glass content, and low ionic diffusion, all of which result in the formation of a porous polycrystalline conglomerate. Further, Figure 5 shows that oxide additions, such as $\alpha-\mathrm{Fe}_{2} \mathrm{O}_{3}$, offer the potential to achieve percolation with higher $\mathrm{Al}_{2} \mathrm{O}_{3}$ contents $(40 \mathrm{wt} \%$ ) approaching that of mullite (48 $\mathrm{wt} \% \mathrm{Al}_{2} \mathrm{O}_{3}$ for fly ash 3 ) to achieve a net mullite composition), albeit with slower kinetics to achieve percolation but at a lower residual glass content.

The selection of the type and amount of additive to enhance the kinetics of percolation will be dependent on the nature of the fly ash (especially its $\mathrm{Al}_{2} \mathrm{O}_{3}: \mathrm{SiO}_{2}$ ratio), the fluxing effectiveness of the oxide addition, the soak temperature, and the soak time. Examination of these variables has the potential to (1) increase the kinetics of percolation by reducing the temperature and/or time required for percolation or (2) reduce the kinetics of percolation while decreasing the amount of residual glass in the triple points.

As discussed previously, the major advantage of percolated mullite over conventional mullite products is the microstructure consisting of a direct-bonded, single-crystal, continuous, needle network that prevents high-temperature deformation and isolates the residual glass in the triple points. These characteristics allow percolated mullite to exhibit unprecedented thermal stability to at least at $1600{ }^{\circ} \mathrm{C}$, as shown by the diametral shrinkage data in Figures 3-5 and the thermal expansion data in Figure 6. In principle, if slip systems are not activated to cause high-temperature deformation, the performance may extend as high as the melting point of mullite $\left(1850^{\circ} \mathrm{C}\right)$.

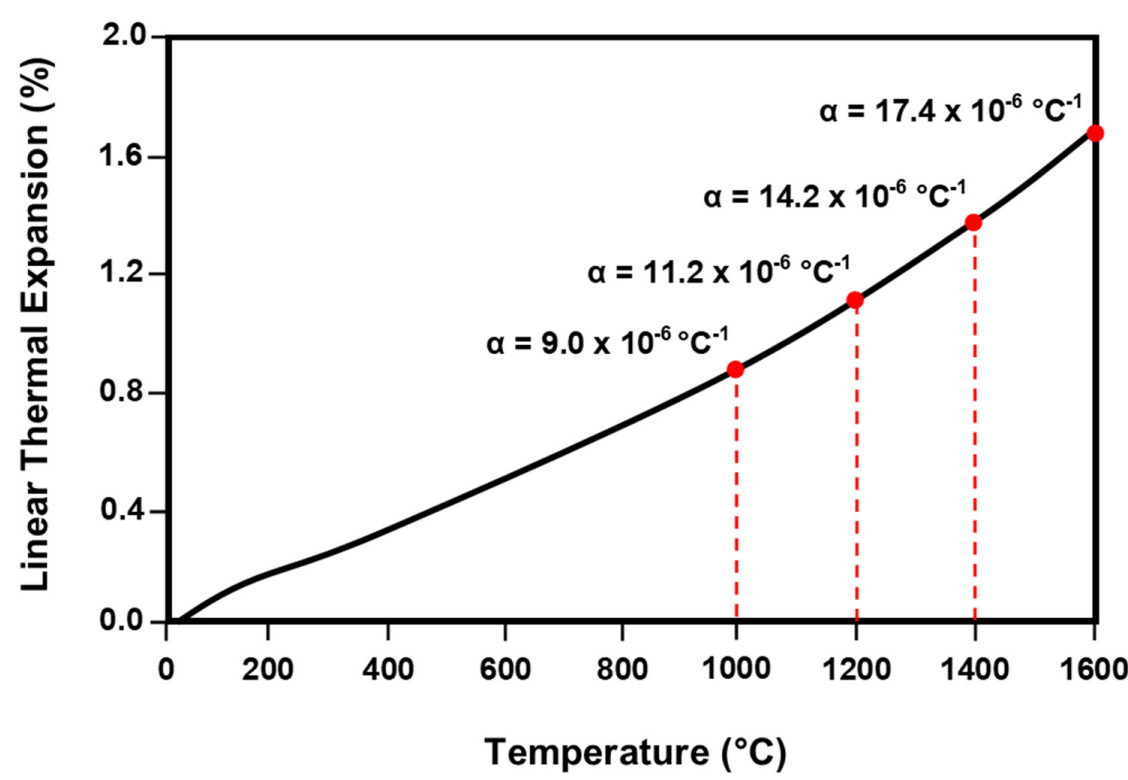

Figure 6. Optical dilatometry data for thermal expansion of percolated mullite from fly ash 2.

These data suggest that the thermal expansion is dominated by the [010] and [001] axial directions of the mullite needles. The expansion at $1000{ }^{\circ} \mathrm{C}$, which is higher than 
the reported bulk expansion of $\alpha_{20-1000}=6.74 \times 10^{-6}{ }^{\circ} \mathrm{C}^{-1}$, may derive from the additive effect of the residual glass in the triple points but it also may be due to differences in the mullite samples and/or the measurement techniques.

These microstructures also can be tailored to exhibit specific properties. At high porosities, the microstructures can be engineered to moderate the low thermal conductivities and low compressive strengths. At low porosities, highly dense microstructures containing only closed spherical pores can be engineered [56] for low thermal conductivities but high compressive strengths [63]. Furthermore, manipulation of the microstructure can be done to create highly dense bodies with small closed spherical pores which help to also lower the thermal conductivity.

Consequently, percolated mullite has been used to fabricate dense and porous sintered refractory shapes, aggregates, and porous refractory shapes from sintered aggregates fabricated from fly ash and oxide additions [56]. These refractory shapes exhibited the physical properties described in Table 4.

Table 4. Physical properties of refractory shapes fabricated from percolated mullite.

\begin{tabular}{ccc}
\hline Refractory & Bulk Density $\mathbf{( k g \cdot \mathbf { m } ^ { - 3 } )}$ & Apparent Porosity (\%) \\
\hline Dense and Porous Shapes & $\sim 1600-2000$ & $\sim 5-30$ \\
Sintered Aggregates & $\sim 900-1100$ & $\sim 40-50$ \\
\hline
\end{tabular}

\section{Summary}

The present work considers previous work on the fabrication of mullite from fly ash and introduces a novel form of volumetrically percolated mullite with microstructures consisting of direct-bonded, single-crystal, continuous, needle networks, with the residual glass isolated in the triple points. These unique microstructures exhibit unprecedented longterm high-temperature dimensional stabilities upon long-term heating at $1500-1600^{\circ} \mathrm{C}$ and possibly higher temperatures. Percolated mullite can be fabricated from both pure fly ash and fly ash with oxide addition, enabling the engineering of the kinetics of percolation to moderate the thermal conductivities and compressive strengths. The application of these novel materials as acidic and neutral refractories has both economic and environmental advantages as they have the potential to utilise high volumes of fly ash, which is a low cost industrial-by product.

Author Contributions: Conceptualization, P.K., S.A.K. and C.C.S.; methodology, P.K. and C.C.S.; validation, P.K.; investigation, P.K., N.H., V.Z., L.S.; resources, P.K., S.A.K., E.J.S. and C.C.S.; writingoriginal draft preparation, P.K., N.H., and V.Z.; writing—review and editing, P.K. and C.C.S.; supervision, P.K., S.A.K., E.J.S. and C.C.S.; project administration, P.K., S.A.K., E.J.S. and C.C.S.; funding acquisition, P.K., S.A.K., E.J.S., and C.C.S. All authors have read and agreed to the published version of the manuscript.

Funding: This research was funded by Vecor Pty. Ltd. (now Vecor Technologies Pty. Ltd.) through direct research funding and through the Australian Research Council Linkage Program (ARC-LP, grant LP150101081).

Institutional Review Board Statement: Not Applicable.

Informed Consent Statement: Not Applicable.

Data Availability Statement: Not Applicable.

Acknowledgments: The authors acknowledge the long-term financial support from Vecor Pty. Ltd. (now Vecor Technologies Pty. Ltd.), funding through the Australian Research Council Linkage Program (ARC-LP, grant LP150101081), and the subsidised characterisation facilities at the Mark Wainwright Analytical Centre, UNSW Sydney, Australia.

Conflicts of Interest: The authors declare no conflict of interest. 


\section{References}

1. Li, J.; Zhuang, X.; Querol, X.; Font, O.; Moreno, N. A Review on the Applications of Coal Combustion Products in China. Int. Geol. Rev. 2018, 60, 671-716. [CrossRef]

2. Fisher, G.L.; Chang, D.P.Y.; Brummer, M. Fly Ash Collected from Electrostatic Precipitators: Microcrystalline Structures and the Mystery of the Spheres. Science 1976, 192, 553-555. [CrossRef] [PubMed]

3. Bhatt, A.; Priyadarshini, S.; Mohankrishnan, A.A.; Abri, A.; Sattler, M.; Techapaphawit, S. Physical, Chemical, and Geotechnical Properties of Coal Fly Ash: A Global Review. Case Stud. Constr. Mater. 2019, 11, e00263. [CrossRef]

4. Ash Development Association of Australia. Coal Combustion Products Handbook, 2nd ed.; Ward, C., Heidrich, C., Yeatman, O., Eds.; HBM Group: Port Kembla, NSW, Australia, 2014.

5. Qadir, S.U.; Raja, V.; Siddiqui, W.A. Morphological and Biochemical Changes in Azadirachta Indica from Coal Combustion Fly Ash Dumping Site from a Thermal Power Plant in Delhi, India. Ecotoxicol. Environ. Saf. 2016, 129, 320-328. [CrossRef]

6. Papadakis, V.G. Effect of Fly Ash on Portland Cement Systems: Part O: Low-Calcium Fly Ash. Cem. Concr. Res. 1999, 29, 1727-1736. [CrossRef]

7. Ahmaruzzaman, M. A Review on the Utilization of Fly Ash. Prog. Energy Combust. Sci. 2010, 36, 327-363. [CrossRef]

8. ASTM C618-05. Standard Specification for Coal Fly Ash and Raw or Calcined Natural Pozzolan for Use in Concrete; ASTM International: West Conshohocken, PA, USA, 2005.

9. Schneider, H.; Schreuer, J.; Hildmann, B. Structure and Properties of Mullite-A Review. J. Eur. Ceram. Soc. 2008, 28, 329-344. [CrossRef]

10. Aramaki, S.; Roy, R. Revised Phase Diagram for the System $\mathrm{Al}_{2} \mathrm{O}_{3}-\mathrm{SiO}_{2}$. J. Am. Ceram. Soc. 1962, 45, 229-242. [CrossRef]

11. Parvizi-Majidi, A. 1/06-Whiskers and Particulates. In Comprehensive Composite Materials; Kelly, A., Zweben, C.H., Eds.; Elsevier: Amsterdam, The Netherlands, 2000; Volume 1, pp. 175-198.

12. Schneider, H.; Komarneni, S. Mullite; Wiley-VCH Verlag GmbH \& Co.: Weinheim, Germany, 2005.

13. Morrell, R. Handbook of Properties of Technical E Engineering Ceramics. Part 1: An Introduction for the Engineer and Designer; HMSO: London, UK, 1989.

14. Ward, C.R.; French, D. Determination of Glass Content and Estimation of Glass Composition in Fly Ash using Quantitative X-Ray Diffractometry. Fuel 2006, 85, 2268-2277. [CrossRef]

15. Roeder, P.L.; Glasser, F.P.; Osborn, E.F. The System $\mathrm{Al}_{2} \mathrm{O}_{3}-\mathrm{Cr}_{2} \mathrm{O}_{3}-\mathrm{SiO}_{2}$. J. Am. Ceram. Soc. 1968, 51, 585-593. [CrossRef]

16. Katsuki, H.; Furuta, S.; Ichinose, H.; Nakao, H. Preparation and Some Properties of Porous Ceramics Sheet Composed of Needle-Like Mullite. J. Ceram. Soc. Jpn. 1988, 96, 1081-1086. [CrossRef]

17. Kim, B.M.; Cho, Y.K.; Yoon, S.Y.; Stevens, R.; Park, H.C. Mullite Whiskers Derived from Kaolin. Ceram. Int. 2009, 35, 579-583. [CrossRef]

18. Abdullayev, A.; Zemke, F.; Gurlo, A.; Bekheet, M.F. Low-Temperature Fluoride-Assisted Synthesis of Mullite Whiskers. RSC Adv. 2020, 10, 31180-31186. [CrossRef]

19. Kriven, W.M.; Palko, J.W.; Sinogeikin, S.; Bass, J.D.; Sayir, A.; Brunaue, G.; Boysen, H.; Frey, F.; Schneider, J. High Temperature Single Crystal Properties of Mullite. J. Eur. Ceram. Soc. 1999, 19, 2529-2541. [CrossRef]

20. Nishikawa, A. Technology of Monolithic Refractories; Plibrico Japan Company Limited: Tokyo, Japan, 1984.

21. Chesters, J.H. Refractories: Production and Properties; Iron and Steel Institute: London, UK, 1973.

22. Orton Ceramic. Cone Equivalent Temperatures. Available online: https://www.ortonceramic.com/files/2676/File/coneequivalent-temperatures-C.pdf (accessed on 8 January 2021).

23. Carniglia, S.C.; Barna, G.L. Handbook of Industrial Refractories Technology: Principles, Types, Properties and Applications; Noyes Publications: Park Ridge, NJ, USA, 1992.

24. Koshy, P.; Gupta, S.; Sahajwalla, V.; Edwards, P. Effect of Silica on High-Temperature Interfacial Phenomena of Monolithic Refractories with Al-Alloy. Metall. Mater. Trans. B 2008, 39, 331-339. [CrossRef]

25. Adabifiroozjaei, E.; Koshy, P.; Sorrell, C.C. Effects of $\mathrm{AlPO}_{4}$ Addition on the Corrosion Resistance of Andalusite-Based LowCement Castables with Molten Al-Alloy. J. Eur. Ceram. Soc. 2013, 33, 1067-1075. [CrossRef]

26. Adabifiroozjaei, E.; Saidi, A.; Monshi, A.; Koshy, P. Effect of Different Calcium Compounds on the Corrosion Resistance of Andalusite-Based Low-Cement Castables in Contact with Molten Al-Alloy. Metall. Mater. Trans. B 2011, 42, 400-411. [CrossRef]

27. Koshy, P.; Gupta, S.; Sahajwalla, V.; Edwards, P. Effect of $\mathrm{CaF}_{2}$ on Interfacial Phenomena of High Alumina Refractories with Al-Alloy. Metall. Mater. Trans. B 2008, 39, 603-611. [CrossRef]

28. Adabifiroozjaei, E.; Koshy, P.; Sorrell, C.C. Effects of $\mathrm{V}_{2} \mathrm{O}_{5}$ Addition on the Corrosion Resistance of Andalusite Based Low-Cement Castables with Molten Al-Alloy. J. Eur. Ceram. Soc. 2012, 32, 1463-1471. [CrossRef]

29. Adabifiroozjaei, E.; Koshy, P.; Sorrell, C.C. Effects of Different Boron Compounds on the Corrosion Resistance of Andalusite-Based Low Cement Castables in Contact with Molten Al-Alloy. Metall. Mater. Trans. B 2012, 43, 5-13. [CrossRef]

30. Sorrell, C.C.; Koshy, P.; Koszo, S. Percolated Mullite and a Method of Forming Same. U.S. Patent 9,527,775, 27 December 2016.

31. Dong, Y.; Feng, X.; Feng, X.; Ding, Y.; Liu, X.; Meng, G. Preparation of Low-Cost Mullite Ceramics from Natural Bauxite and Industrial Waste Fly Ash. J. Alloys Compd. 2008, 460, 599-606. [CrossRef]

32. Park, Y.M.; Yang, T.Y.; Yoon, S.Y.; Stevens, R.; Park, H.C. Mullite Whiskers Derived from Coal Fly Ash. Mater. Sci. Eng. A 2007, 454-455, 518-522. [CrossRef]

33. Cao, J.; Dong, X.; Li, L.; Dong, Y.; Hampshire, S. Recycling of Waste Fly Ash for Production of Porous Mullite Ceramic Membrane Supports with Increased Porosity. J. Eur. Ceram. Soc. 2014, 34, 3181-3194. [CrossRef] 
34. Dong, Y. Recycling of Fly Ash for Preparing Porous Mullite Membrane Supports with Titania Addition. J. Hazard. Mater. 2010, 180, 173-180. [CrossRef] [PubMed]

35. Li, S.; Du, H.; Guo, A.; Xu, H.; Yang, D. Preparation of Self-Reinforcement of Porous Mullite Ceramics Through In Situ Synthesis of Mullite Whisker in Flyash Body. Ceram. Int. 2012, 38, 1027-1032. [CrossRef]

36. Li, J.-H.; Ma, H.-W.; Huang, W.-H. Effect of $\mathrm{V}_{2} \mathrm{O}_{5}$ on the Properties of Mullite Ceramics Synthesized from High-Aluminum Fly Ash and Bauxite. J. Hazard. Mater. 2009, 166, 1535-1539. [CrossRef]

37. Chen, A.-N.; Li, M.; Xu, J.; Lou, C.-H.; Wu, J.; Cheng, L.; Shi, Y.; Li, C. High-Porosity Mullite Ceramic Foams Prepared by Selective Laser Sintering using Fly Ash Hollow Spheres as Raw Materials. J. Eur. Ceram. Soc. 2018, 38, 4553-4559. [CrossRef]

38. Dong, Y.; Hampshire, S.; Zhou, J.; Li, Z.; Wang, J.; Meng, G. Sintering and Characterization of Flyash-Based Mullite with MgO Addition. J. Eur. Ceram. Soc. 2011, 31, 687-695. [CrossRef]

39. Guo, A.; Liu, J.; Xu, R.; Xu, H.; Wang, C. Preparation of Mullite from Desilication-Flyash. Fuel 2010, 89, 3630-3636. [CrossRef]

40. Lin, B.; Li, S.; Hou, X.; Li, H. Preparation of High Performance Mullite Ceramics from High-Aluminum Fly Ash by an Effective Method. J. Alloys Compd. 2015, 623, 359-361. [CrossRef]

41. Ma, B.; Su, C.; Ren, X.; Qian, F.; Yang, W.; Liu, G.; Li, H.; Yu, J.; Zhu, Q. Preparation and Properties of Porous Mullite Ceramics with High-Closed Porosity and High Strength from Fly Ash via Reaction Synthesis Process. J. Alloys Compd 2019, 803, 981-991. [CrossRef]

42. Das, D.; Nijhuma, K.; Gabriel, A.M.; Daniel, G.P.F.; de Murilo, D.M.I. Recycling of Coal Fly Ash for Fabrication of Elongated Mullite Rod Bonded Porous SiC Ceramic Membrane and its Application in Filtration. J. Eur. Ceram. Soc. 2020, 40, 2163-2172. [CrossRef]

43. Li, Y.; Lu, J.; Zeng, Y.; Liu, Z.; Wang, C. Preparation and Characterization of Mullite Powders from Coal Fly Ash by the Mullitization and Hydrothermal Processes. Mater. Chem. Phys. 2018, 213, 518-524. [CrossRef]

44. Fu, M.; Liu, J.; Dong, X.; Zhu, L.; Dong, Y.; Hampshire, S. Waste Recycling of Coal Fly Ash for Design of Highly Porous Whisker-Structured Mullite Ceramic Membranes. J. Eur. Ceram. Soc. 2019, 39, 5320-5331. [CrossRef]

45. Luo, Y.; Ma, S.; Zheng, S.; Liu, C.; Han, D.; Wang, X. Mullite-Based Ceramic Tiles Produced Solely from High-Alumina Fly Ash: Preparation and Sintering Mechanism. J. Alloys Compd. 2018, 732, 828-837. [CrossRef]

46. Li, C.; Zhou, Y.; Tian, Y.; Zhao, Y.; Wang, K.; Li, G.; Chai, Y. Preparation and Characterization of Mullite Whisker Reinforced Ceramics Made from Coal Fly Ash. Ceram. Int. 2019, 45, 5613-5616. [CrossRef]

47. Tan, H. Preparation of Mullite Whiskers from Coal Fly Ash using Sodium Sulfate Flux. Int. J. Miner. Process. 2011, 100, 188-189. [CrossRef]

48. Chen, X.; Li, T.; Ren, Q.; Wu, X.; Li, H.; Dang, A.; Zhao, T.; Shang, Y.; Zhang, Y. Mullite Whisker Network Reinforced Ceramic with High Strength and Lightweight. J. Alloys Compd. 2017, 700, 37-42. [CrossRef]

49. Wang, T.; Ma, S.; Wang, X.; Hong, T.; Luo, Y. A 100\% High-Aluminum Fly Ash-Based High-Density Mullite Ceramic with a Triple Microstructure: Preparation and Mechanical Characterization. Constr. Build. Mater. 2020, 239, 117761. [CrossRef]

50. Ren, S.; Tao, X.; Xu, X.; Guo, A.; Liu, J.; Fan, J.; Ge, J.; Fang, D.; Liang, J. Preparation and Characteristic of the Fly Ash Cenospheres/Mullite Composite for High-Temperature Application. Fuel 2018, 233, 335-345. [CrossRef]

51. Chen, J.; Shao, L.; Lu, J. Synthesis of Mullite from High-Alumina Fly Ash: A Case from the Jungar Power Plant in Inner Mongolia, Northern China. Acta Geol. Sin. Engl. Ed. 2008, 82, 99-104.

52. Han, G.; Yang, S.; Peng, W.; Huang, Y.; Wu, H.; Chai, W.; Liu, J. Enhanced Recycling and Utilization of Mullite from Coal Fly Ash with a Flotation and Metallurgy Process. J. Clean. Prod. 2018, 178, 804-813. [CrossRef]

53. Foo, C.T.; Salleh, M.A.M.; Ying, K.K.; Matori, K.A. Mineralogy and Thermal Expansion Study of Mullite-Based Ceramics Synthesized From Coal Fly Ash and Aluminum Dross Industrial Wastes. Ceram. Int. 2019, 45, 7488-7494. [CrossRef]

54. Yadav, A.K.; Patel, S.; Bhattacharyya, S. Preparation of Low-Cost Porous Mullite Ceramics by Recycling Fly Ash. AIP Conf. Proc. 2019, 2142, 30004.

55. Zhu, J.; Yan, H. Microstructure and Properties of Mullite-Based Porous Ceramics Produced from Coal Fly Ash with $\mathrm{Added}_{2} \mathrm{Al}_{3}$. Int. J. Miner. Metall. Mater. 2017, 24, 309-315. [CrossRef]

56. Koshy, P.; Koszo, S.A.; Severin, E.; Sorrell, C.C. High-Performance Refractory Ceramics of Percolated Mullite from Waste Materials. Am. Ceram. Soc. Bull. 2018, 97, 29-33.

57. Adabifiroozjaei, E.; Hart, J.N.; Koshy, P.; Mitchell, D.R.G.; Sorrell, C.C. Mullite-Glass and Mullite-Mullite Interfaces: Analysis by Molecular Dynamics (MD) Simulation and High-Resolution TEM. J. Am. Ceram. Soc. 2017, 101, 428-439. [CrossRef]

58. Uchino, K. Piezoelectric Composite Materials. In Reference Module in Materials Science and Materials Engineering; Hashmi, S., Ed.; Elsevier: Oxford, UK, 2016; pp. 1-12.

59. Newnham, R.E.; Skinner, D.P.; Cross, L.E. Connectivity and Piezoelectric-Pyroelectric Composites. Mater. Res. Bull. 1978, 13, 525-536. [CrossRef]

60. Levin, E.M.; Robbins, C.R.; McMurdie, H.F. (Eds.) Figures 630, 696, and 712. In Phase Diagrams for Ceramists; American Ceramic Society: Columbus, OH, USA, 1964.

61. Jak, E.; Hayes, P.; Pelton, A.; Dectorov, S. Thermodynamic Modelling of the $\mathrm{Al}_{2} \mathrm{O}_{3}-\mathrm{CaO}-\mathrm{FeO}-\mathrm{Fe}_{2} \mathrm{O}_{3}-\mathrm{PbO}-\mathrm{SiO}{ }_{2}-\mathrm{ZnO}$ System with Addition of $\mathrm{K}$ and Na with Metallurgical Applications. In Proceedings of the VIII International Conference on Molten Slags, Fluxes and Salts-MOLTEN 2009, Santiago, Chile, 18-21 January 2009; Sánchez, M., Parra, R., Riveros, G., Diaz, C., Eds.; Gecamin Ltd.: Santiago, Chile, 2009; pp. 473-490.

62. Brownell, W.E. Structural Clay Products; Springer-Verlag: Vienna, Austria, 1976.

63. Kingery, W.D.; Bowen, H.K.; Uhlmann, D.R. Introduction to Ceramics, 2nd ed.; John Wiley: New York, NY, USA, 1976. 